\title{
Data transmission through optical polarization domain walls
}

\author{
M. Guasoni ${ }^{1,3}$, M. Gilles ${ }^{1}$, P-Y. Bony ${ }^{1}$, J. Garnier ${ }^{2}$, A. Picozzi ${ }^{1}$ and J. Fatome ${ }^{1, *}$ \\ ${ }^{1}$ Laboratoire Interdisciplinaire Carnot de Bourgogne (ICB), UMR 6303 CNRS - Université Bourgogne Franche-Comté, \\ 9 Avenue Alain Savary, BP 47870, 21078 Dijon, France \\ ${ }^{2}$ Laboratoire de Probabilités et Modèles Aléatoires, University of Paris VII, 75251 Paris, France \\ ${ }^{3}$ Optoelectronics Research Centre, University of Southampton, SO17 1BJ, United Kingdom. \\ *Corresponding author: jfatome@u-bourgogne.fr
}

Optical domain walls (DW) correspond to a localized structure of the kink type that connects two regions of space with different polarizations [1]. In such a symbiotic system, the fast polarization knots lead to two anticorrelated coupled twin-waves for which the strong binding force imposed by cross-phase modulation (XPM) can compensate for the classical defocusing regime. In this contribution, we report a direct observation of polarization domain walls (PDWs) propagating within a standard optical fiber commonly used in optical communications [2]. We exploit their topological properties to overcome the nonlinear Kerr-limits usually imposed in normally dispersive fibers for optical data transmission.

In order to generate a periodic train of PDWs, a 1555-nm continuous-wave was first intensity modulated by 30-ps square-shape pulses at a repetition rate of $14 \mathrm{GHz}$. This pulse train is then split in 2 out-of-phase, delayed and polarization multiplexed replica so as to obtain a pure orthogonal polarization flip-flopping at 28-GHz. After amplification, these PDWs are injected into a 10-km long normally dispersive standard fiber (TWHD from ofs D $=-15 \mathrm{ps} / \mathrm{nm} / \mathrm{km}$ at $1555 \mathrm{~nm}$ ). Fig. 1a displays the resulting 28-GHz signal after $10 \mathrm{~km}$ of propagation for an input power of $22 \mathrm{dBm}$ per polarization component. At first, when only one polarization component is injected into the fiber (black dotted-line), due to combined effects of chromatic dispersion and self-phase modulation, the output signal rapidly deteriorates into a complex periodic pattern. In contrast, when both orthogonally polarized twinwaves propagate along the fiber (red \& blue solid-lines), the XPM effect acts in full strength leading to an undistorted propagation of PDWs. In order to confirm that the system propagates genuine kink solitons, we have assessed the capability of PDWs to transmit optical data. For this proof-of-principle, we propagate a 10-Gbit/s binary sequence corresponding to the ASCII code of the ERC project acronym PETAL. The encoded PDWs propagate in a first length of $25-\mathrm{km}$ of TWHD fiber before reamplification and transmission in a second span of $25 \mathrm{~km}$. Figure $1 \mathrm{~b}$ illustrates the PETAL sequence monitored after $50 \mathrm{~km}$ of propagation as a function of the transmitted power when only one of the two twin-waves is injected. In this case, we clearly observe a significant signal degradation leading to a complete loss of the data. In contrast, when both twin-waves propagate simultaneously (Fig. 1c), we can clearly observe that the energy remains efficiently locked within each domain and consequently that the data sequence is ideally preserved. These observations confirm the capacity of PDWs to be addressed individually as well as the solitary nature of such kink structures.
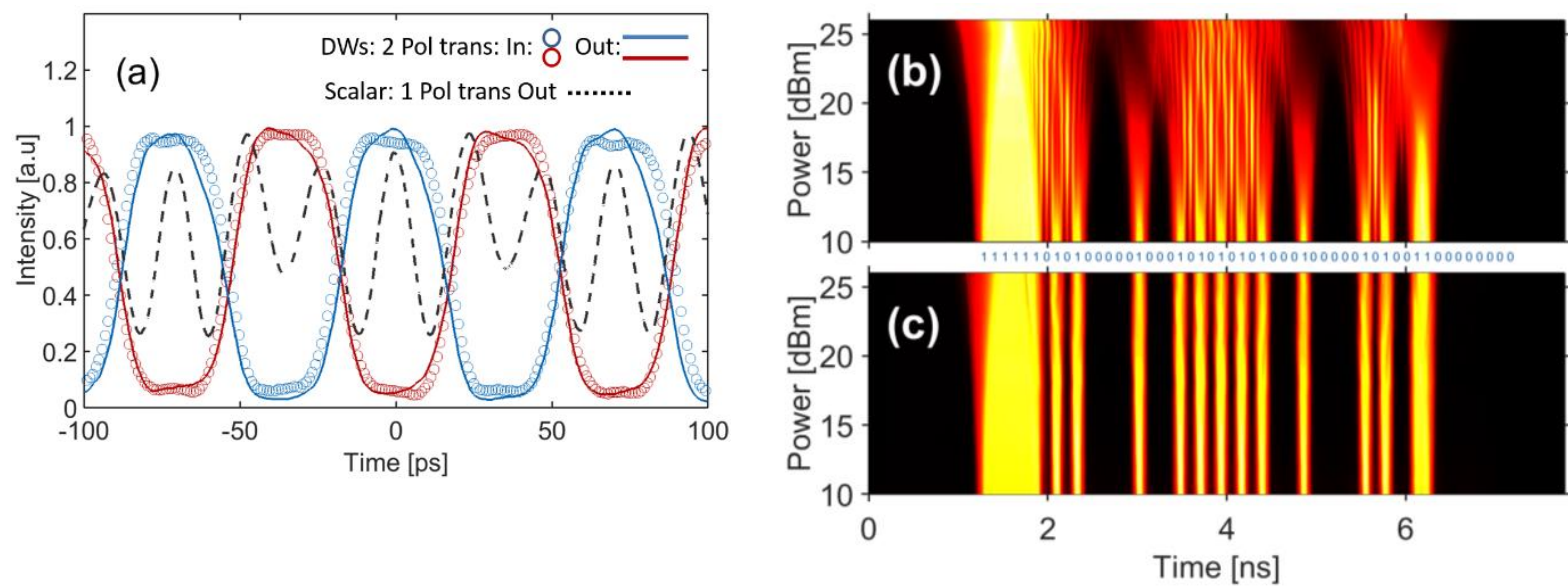

Fig. 1 (a) Output intensity profiles for a 28-GHz PDWs propagation: 1 polarization transmitted: dotted-line, both polarizations injected: solid-lines, input signal is shown in circles (b-c) 10-Gbit/s PDWs transmission: (b) 1 polarization transmitted, (c) both polarizations injected.

\section{References}

[1] M. Haelterman and A. P. Sheppard, "Polarization domain walls in diffractive or dispersive Kerr media," Opt. Lett. 19, 96-98 (1994).

[2] M. Gilles et al. "Polarization domain walls in optical fibres as topological bits for data transmission," Nat. Photon. in press (2017). 